Research Article

\title{
Hospital Mortality and Resource Implications of Hospitalisation with COVID-19 in London, UK: A Prospective Cohort Study
}

\author{
Savvas Vlachos (D), ${ }^{1}$ Adrian Wong, ${ }^{1}$ Victoria Metaxa, ${ }^{1}$ Sergio Canestrini, ${ }^{1,2}$ \\ Carmen Lopez Soto, ${ }^{1}$ Jimstan Periselneris, ${ }^{3}$ Kai Lee, ${ }^{3}$ Tanya Patrick, ${ }^{3}$ Christopher Stovin, ${ }^{3}$ \\ Katrina Abernethy, ${ }^{3}$ Budoor Albudoor, ${ }^{1}$ Rishi Banerjee, ${ }^{1}$ Fatimah Juma, \\ Sara Al-Hashimi, ${ }^{1}$ William Bernal, ${ }^{1}$ and Ritesh Maharaj (iD ${ }^{1,4,5}$ \\ ${ }^{1}$ Department of Critical Care Medicine, King's College Hospital NHS Foundation Trust, Denmark Hill, SE5 9RS, London, UK \\ ${ }^{2}$ Centre for Social Ethics and Policy, Department of Law, School of Social Science, The University of Manchester, Oxford Road, \\ M13 9PL, Manchester, UK \\ ${ }^{3}$ Department of Respiratory Medicine, King's College Hospital NHS Foundation Trust, Denmark Hill, SE5 9RS, London, UK \\ ${ }^{4}$ School of Health and Social Care Research, King's College London, Strand, WC2R 2LS, London, UK \\ ${ }^{5}$ Department of Health Policy, London School of Economics, Houghton St, Holborn, WC2A 2AE, London, UK
}

Correspondence should be addressed to Savvas Vlachos; savvas.vlachos@nhs.net

Received 13 August 2020; Accepted 30 December 2020; Published 29 January 2021

Academic Editor: Thomas Esposito

Copyright ( $\odot 2021$ Savvas Vlachos et al. This is an open access article distributed under the Creative Commons Attribution License, which permits unrestricted use, distribution, and reproduction in any medium, provided the original work is properly cited.

Background. Coronavirus disease 2019 (COVID-19) had a significant impact on the National Health Service in the United Kingdom (UK), with over 35000 cases reported in London by July 30, 2020. Detailed hospital-level information on patient characteristics, outcomes, and capacity strain is currently scarce but would guide clinical decision-making and inform prioritisation and planning. Methods. We aimed to determine factors associated with hospital mortality and describe hospital and ICU strain by conducting a prospective cohort study at a tertiary academic centre in London, UK. We included adult patients admitted to the hospital with laboratory-confirmed COVID-19 and followed them up until hospital discharge or 30 days. Baseline factors that are associated with hospital mortality were identified via semiparametric and parametric survival analyses. Results. Our study included 429 patients: $18 \%$ of them were admitted to the ICU, $52 \%$ met criteria for ICU outreach team activation, and $61 \%$ had treatment limitations placed during their admission. Hospital mortality was $26 \%$ and ICU mortality was $34 \%$. Hospital mortality was independently associated with increasing age, male sex, history of chronic kidney disease, increasing baseline C-reactive protein level, and dyspnoea at presentation. COVID-19 resulted in substantial ICU and hospital strain, with up to 9 daily ICU admissions and 41 daily hospital admissions, to a peak census of 80 infected patients admitted in the ICU and 250 in the hospital. Management of such a surge required extensive reorganisation of critical care services with expansion of ICU capacity from 69 to 129 beds, redeployment of staff from other hospital areas, and coordinated hospital-level effort. Conclusions. COVID19 is associated with a high burden of mortality for patients treated on the ward and the ICU and required substantial reconfiguration of critical care services. This has significant implications for planning and resource utilisation.

\section{Introduction}

Coronavirus disease 2019 (COVID-19), an infectious syndrome caused by SARS-CoV-2, appeared in December 2019 and evolved into a pandemic that caused more than eleven million cases and 530000 deaths worldwide by July 2020 [1]. The high number and acuity of patients resulted in an unprecedented demand for hospitalisation and critical care services in many affected countries. Developed areas such as Wuhan (China), Lombardy (Italy), and New York (United 
States of America) reported a surge in critically ill patients, which quickly led to significant strain on healthcare systems through shortages in the Intensive Care Unit (ICU) beds, equipment, and trained personnel [2-4].

There was concern that the United Kingdom (UK) would face similar challenges, particularly in densely populated areas like London. The first infection was reported on January 30, 2020, and by late July, the country had recorded a large number of cases, with over 35000 confirmed infections in the greater area of London alone [5].

Despite the high number of hospital admissions with COVID-19, the existing peer-reviewed literature in the UK remains restricted to large population-level studies and small retrospective cohorts with few details on clinical management and hospital-level strain [6-8]. Major reports published so far focus either on large but incomplete hospital cohorts with missing data and short follow-up time [7] or exclusively on ICU admissions [8] with no connection between the two populations. Extrapolation from international settings is difficult due to significant differences in population characteristics [2], health system organisation $[2,4,9]$, and perceived health system strain [10].

Detailed local patient-level information on characteristics and outcomes, as well as institution-level information on service pressures, would guide clinical decision-making and inform effective prioritisation and resource allocation in the future, particularly in circumstances when a surge in demand places the National Health Service again at risk of being overwhelmed. The aim of our study was to describe the clinical characteristics and course of hospitalised patients with COVID-19, as well as its broader resource implications for a tertiary academic hospital in London, UK.

\section{Methods}

2.1. Study Design. We conducted a prospective cohort study at King's College Hospital (KCH), a tertiary academic centre in London, UK. Institutional (IRAS-256619; April 15, 2020) and regional (Health Research Authority; IRAS-256619; April 22, 2020) review board approvals waived the need for ethics committee review and the need for informed patient consent.

All consecutive patients tested for SARS-CoV-2 infection using reverse-transcriptase polymerase chain reaction (RT-PCR) assays of respiratory tract samples between February 25 and March 31, 2020, were considered eligible for study participation. Inclusion criteria were age of 18 years or above and laboratory-confirmed SARS-CoV-2 infection, which was defined as at least one positive RT-PCR nasopharyngeal swab [11]. Patients with missing identifiers and missing SARS-CoV-2 test results and those transferred from other hospitals were excluded. Included patients were followed up until death, hospital discharge, or 30 days after hospital admission. Follow-up was concluded on April 30, 2020. For patients with multiple hospital or ICU admissions, only the first admission was recorded. Transfers between different ICUs within the hospital were considered part of the same admission. The primary outcome was mortality at hospital discharge or at 30 days. Secondary outcomes were
ICU mortality, as well as hospital and ICU capacity strain, measured in bed occupancy. The report of our findings is based on the Strengthening the Reporting of Observational Studies in Epidemiology (STROBE) Statement [12]. More details regarding the institutional setting and pandemic surge are available in Supplementary File 1.

2.2. Data Collection and Management. Trained members of the clinical team extracted anonymised data from electronic health records (EHRs) [13]. We recorded the following for all included patients: age, sex, ethnicity, area-level socioeconomic deprivation (Index of Multiple Deprivation [14]) clinical frailty, medical comorbidities, Age-adjusted Charlson Comorbidity Index (ACCI), body mass index (BMI), prior residence, self-reported presenting symptoms, reason for hospital admission, laboratory, microbiological, and imaging tests, COVID-19-specific drug treatments, treatment limitations such as Treatment Escalation Plans (TEPs) or do-not-resuscitate (DNR) orders, length of hospital stay, and outcome at hospital discharge or at 30 days.

For patients who were initially admitted to the ward, we also recorded peak temperature and National Early Warning Score version 2 (NEWS2) score [15], maximum level of respiratory support, and development of complications such as hypoxia, hypotension, tachycardia, and depressed consciousness. For patients who were admitted to the ICU, we additionally recorded reason for ICU admission, daily Sequential Organ Failure Assessment (SOFA) score [16], organ support (mechanical ventilation, renal replacement therapy, and circulatory support), arterial partial pressure of oxygen $\left(\mathrm{P}_{\mathrm{a}} \mathrm{O}_{2}\right)$, fraction of inspired oxygen $\left(\mathrm{F}_{i} \mathrm{O}_{2}\right), \mathrm{P}_{\mathrm{a}} \mathrm{O}_{2} / \mathrm{F}_{i} \mathrm{O}_{2}$ ratio (PFR), use of medications such as vasopressors, pulmonary vasodilators, and neuromuscular blocking drugs, use of prone positioning or extracorporeal membrane oxygenation (ECMO), insertion of tracheostomy, length of ICU stay, and outcome at ICU discharge or at 30 days.

We recorded comorbidities and symptoms based on electronic case note review and categorised variables according to clinical relevance and current literature $[4,10,17]$. We used only validated laboratory results and official reports of imaging studies. Initial tests for newly admitted COVID-19 patients refer to those performed within 24 hours of hospital admission; for patients already admitted, they refer to tests performed within 24 hours of COVID-19 diagnosis. Clinical frailty was assessed on a 9category scale [18], using information available in the EHR; patients with a score above 4 were considered frail [19]. Treatment escalation plans (TEPs) refer to structured assessments of patients' suitability regarding specific aspects of treatment such as organ support or ICU admission [20]. Acute kidney injury (AKI) was defined according to KidneyDisease Improving Global Outcomes (KDIGO) criteria [21]. More details regarding definitions of collected data are provided in Supplementary File 2.

2.3. Statistical Methods. Descriptive analyses are presented as median (IQR) or number (\%), and we avoided univariate comparisons between groups. To identify factors associated 
with the time to death at hospital discharge or at 30 days, we performed multivariable Cox proportional hazards and parametric survival analyses. Covariate inclusion followed a structured approach. We tested the proportional hazards assumption and investigated interactions of the included covariates with sex and age. We also assessed whether the baseline survival experience differed by categories of age, sex, frailty, and ethnicity, using stratified Cox regression. The effect of each included covariate was quantified by calculating adjusted hazard ratios (HRs) with 95\% confidence intervals $(95 \% \mathrm{CI})$. We followed a similar approach for the parametric analysis and plotted the hazard function over time for six hypothetical patients, in order to show the effect of each included covariate on the hazard of death. Statistical tests were 2-sided, with an $\alpha$-level of 0.05 for statistical significance. We did not impute any missing data. Analyses were performed using Stata/MP version 15.1 (StataCorp). Details regarding the statistical approach are provided in Supplementary File 3.

\section{Results}

Between February 25 and March 31, 2020, 2728 patients were tested for SARS-CoV-2 infection at $\mathrm{KCH}$. After excluding 170 patients (6\%), 2558 patients (94\%) were screened for inclusion; 2129 (83\%) of them did not meet the inclusion criteria, and $429(17 \%)$ were included in the study. Among them, 353 patients (82\%) were treated only on the ward and $76(18 \%)$ were treated in the ICU. The study flow diagram is shown in Figure 1.

Baseline patient characteristics are shown in Table 1. Patients had a median age of 65 years (IQR $52-81$ ), and $61 \%$ were of ethnic minority background. The majority (61\%) were overweight and $90 \%$ of them had at least one serious comorbidity; the most common were hypertension (52\%), diabetes (37\%), and chronic kidney disease (CKD) (14\%). The most common presenting complaints included cough (62\%), fever (62\%), and dyspnoea ( $43 \%$ ), but only $42 \%$ of patients were hospitalised for hypoxaemic respiratory failure. Initial chest $\mathrm{X}$-rays were normal in $23 \%$ of patients and showed diffuse, bilateral infiltrates in more than half.

Compared to ward patients, those admitted to the ICU were younger and less commonly frail and had a lower burden of chronic comorbidities, as described by the ACCI. They were, however, more likely to be diabetic and to be hospitalised for respiratory failure, with significantly more deranged initial laboratory tests and abnormal chest imaging.

Admission laboratory findings for all patients are shown in Table 2. Lymphopenia was common, as were elevations in C-reactive protein (CRP) (median $75 \mathrm{mg} / \mathrm{L}$, IQR 28-143) and creatinine (median $92 \mathrm{mg} / \mathrm{L}, \mathrm{IQR} 68-130$ ). Raised lactate dehydrogenase, ferritin, creatine kinase, and D-dimers were also common, albeit measured in a minority of patients.

3.1. Patients Treated on the Ward. Most patients developed significant morbidity during their hospital admission. Fiftytwo percent met the criteria for critical care outreach team activation (NEWS2 score above six), predominantly for respiratory failure (Table 3 ). On chest computed tomography (CT), more than $70 \%$ of scans showed bilateral diffuse infiltrates and $25 \%$ revealed pulmonary embolism (PE). PE was more frequently identified in ICU patients (33\%). Subsequent laboratory tests revealed worsening lymphopenia, raised CRP (median $159 \mathrm{mg} / \mathrm{L}$, IQR 81-299) and intracellular enzymes, and impaired hepatic, renal, and haemostatic function (Table 2). Overall, $21 \%$ of patients developed KDIGO stage three AKI, but the incidence among ICU patients (67\%) was much higher than that among ward patients $(11 \%)$. We recorded only mild degrees of myocardial involvement, evidenced by small increases in values of high-sensitivity troponin $\mathrm{T}$ and unremarkable echocardiographic findings. Secondary infections were common in the ICU-treated group: $50 \%$ had a positive respiratory tract sample and $41 \%$ had a positive blood culture. Samples from the respiratory tract were frequently positive for Gramnegative organisms and fungi.

Treatment limitations were placed in $61 \%$ of patients overall, most commonly on hospital admission (median 0 days, IQR $0-1$ ). The majority (>80\%) of these limitations involved TEPs and DNR orders. Two-thirds of patients with a TEP were considered frail. Among patients with treatment limitations that were not considered frail, many had significant medical comorbidities such as active malignancy and stroke.

3.2. Patients Treated in ICU. The clinical trajectory of 76 patients who were admitted to the ICU is described in Table 4. Many patients developed precipitous respiratory failure and required ICU admission directly from the emergency department (57\%). Multiple organ failure was common with $97 \%$ requiring mechanical ventilation for hypoxic respiratory failure, $80 \%$ requiring pharmacologic circulatory support, and 57\% requiring renal replacement therapy (RRT) during their ICU stay. The severity of illness was reflected in the high SOFA scores, which remained elevated even after two weeks of ICU stay. The median duration of mechanical ventilation was 12 days (IQR 6-23) and that of renal replacement was 11 days (IQR 4-17). Patients frequently required rescue oxygenation strategies (54\%), which included neuromuscular blocking drugs, inhaled prostacyclin, and prone positioning. Extracorporeal membrane oxygenation (ECMO) was used only in 5\% of ICU patients. A significant proportion (38\%) of patients required a tracheostomy, which was performed at a median of 16.5 days (IQR 14-20) after ICU admission. Almost all laboratory parameters were more deranged among ICU patients, compared to those treated on the ward (Table 2).

3.3. Outcomes. Unadjusted mortality at hospital discharge or at 30 days was $26 \%$ overall, $23 \%$ for ward patients, and $38 \%$ for those treated in the ICU. In unadjusted Cox survival analysis, hospital mortality was associated with age, sex, ACCI score, frailty category, CRP, creatinine, CKD, diabetes, and dyspnoea or fever as presenting complaints. After adjustment, it was independently associated with increasing 


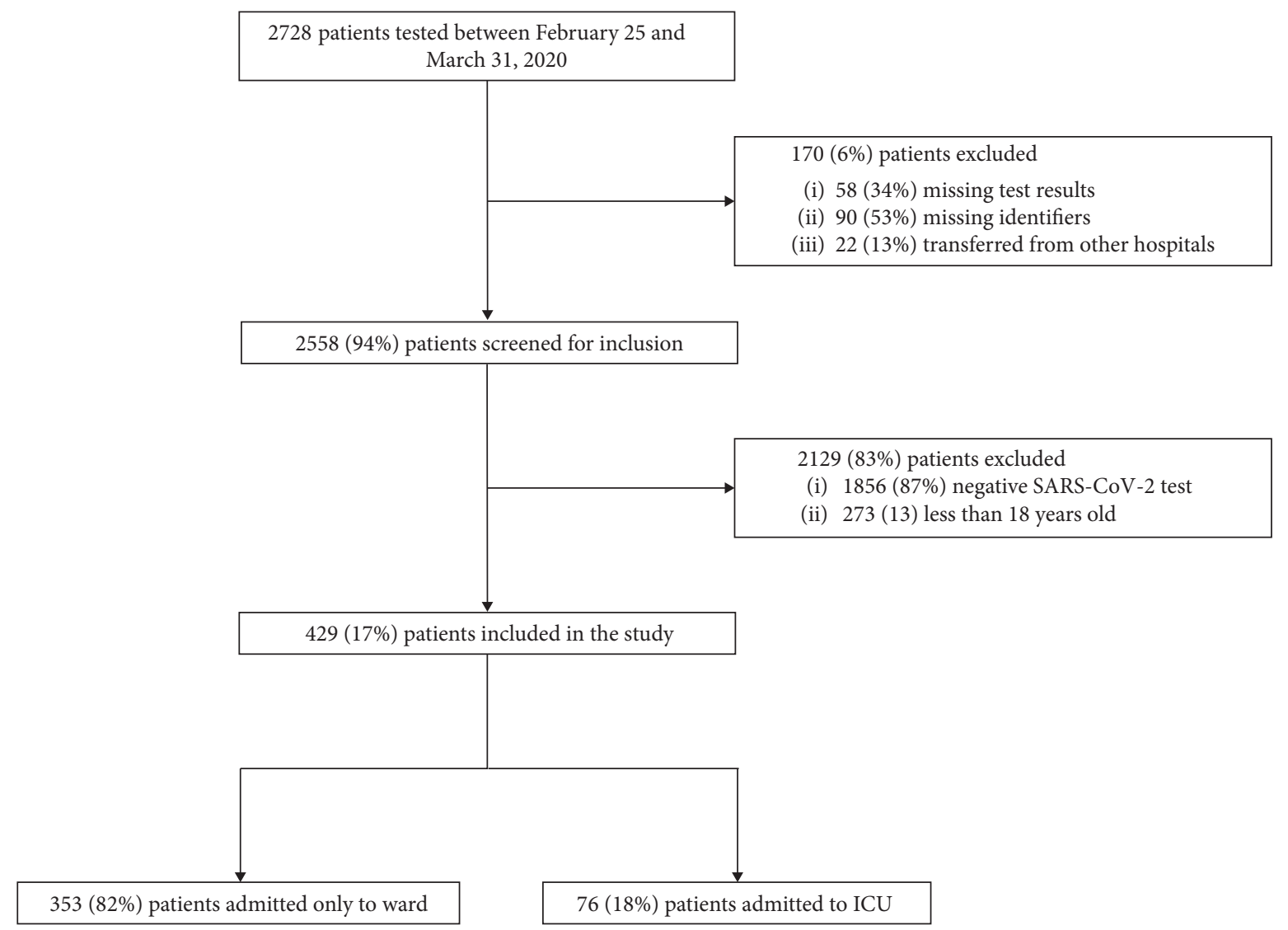

Figure 1: The study flow diagram.

TABLe 1: Baseline characteristics of patients, by admission location.

\begin{tabular}{|c|c|c|c|}
\hline & Study population $(n=429)$ & Treated only on ward $(n=353)$ & Treated in ICU $(n=76)$ \\
\hline Age, years & $65(52-81)$ & $68(54-82)$ & $57(48-63)$ \\
\hline $18-29$ & $12(3 \%)$ & $9(2 \%)$ & $3(4 \%)$ \\
\hline $30-39$ & $27(6 \%)$ & $21(6 \%)$ & $6(8 \%)$ \\
\hline $40-49$ & $37(9 \%)$ & $26(7 \%)$ & $11(14 \%)$ \\
\hline $50-59$ & $79(18 \%)$ & $56(16 \%)$ & $23(30 \%)$ \\
\hline $60-69$ & $90(21 \%)$ & $69(19 \%)$ & $21(28 \%)$ \\
\hline $70-79$ & $64(15 \%)$ & $55(16 \%)$ & $9(12 \%)$ \\
\hline $80-89$ & $82(19 \%)$ & $79(22 \%)$ & $3(4 \%)$ \\
\hline$\geq 90$ & $38(9 \%)$ & $38(11 \%)$ & $0(0 \%)$ \\
\hline \multicolumn{4}{|l|}{ Sex } \\
\hline Female & $195(45 \%)$ & $169(48 \%)$ & $26(34 \%)$ \\
\hline Male & $234(55 \%)$ & $184(52 \%)$ & $50(66 \%)$ \\
\hline \multicolumn{4}{|l|}{ Ethnicity* } \\
\hline Black & $187 / 391(48 \%)$ & $148(47 \%)$ & $39(53 \%)$ \\
\hline Asian & $15 / 391(4 \%)$ & $9(3 \%)$ & $6(8 \%)$ \\
\hline White & $151 / 391(38 \%)$ & $129(40 \%)$ & $22(30 \%)$ \\
\hline Mixed or others & $38 / 391(10 \%)$ & $31(10 \%)$ & $7(9 \%)$ \\
\hline $\mathrm{BMI}^{\dagger}$ & $26.6(23.2-31.2)$ & $26.2(22.8-31.0)$ & $27.8(24.2-32.0)$ \\
\hline$\leq 25$ & $115 / 296(39 \%)$ & $90 / 222(40 \%)$ & $25 / 74(34 \%)$ \\
\hline $26-30$ & $91 / 296(31 \%)$ & $69 / 222(31 \%)$ & $22 / 74(30 \%)$ \\
\hline $21-35$ & $54 / 296(18 \%)$ & $36 / 222(16 \%)$ & $18 / 74(24 \%)$ \\
\hline $36-40$ & $18 / 296(6 \%)$ & $13 / 222(6 \%)$ & $5 / 74(7 \%)$ \\
\hline$\geq 40$ & $18 / 296(6 \%)$ & $14 / 222(6 \%)$ & $4 / 74(5 \%)$ \\
\hline $\mathrm{ACCI}^{\ddagger}$ & $4(2-6)$ & $4(2-6)$ & $2(1-4)$ \\
\hline 0 & $40 / 401(10 \%)$ & $33 / 33(10 \%)$ & $7 / 70(10 \%)$ \\
\hline 1 & $49 / 401(12 \%)$ & $34(10 \%)$ & $15(21 \%)$ \\
\hline
\end{tabular}


TABLE 1: Continued.

\begin{tabular}{|c|c|c|c|}
\hline & Study population $(n=429)$ & Treated only on ward $(n=353)$ & Treated in ICU $(n=76)$ \\
\hline 2 & $51 / 401(13 \%)$ & $37(11 \%)$ & $14(20 \%)$ \\
\hline$\geq 3$ & $261 / 401(65 \%)$ & $227(69 \%)$ & $34(49 \%)$ \\
\hline \multicolumn{4}{|l|}{ IMD quintile } \\
\hline 1 (least deprived) & $9 / 427(2 \%)$ & $6 / 351(2 \%)$ & $3(4 \%)$ \\
\hline 2 & $36 / 427(8 \%)$ & $31 / 351(9 \%)$ & $5(7 \%)$ \\
\hline 3 & $86 / 427(20 \%)$ & $72 / 351(20 \%)$ & $14(18 \%)$ \\
\hline 4 & $204 / 427(48 \%)$ & $167 / 351(48 \%)$ & $37(49 \%)$ \\
\hline 5 (most deprived) & $92 / 427(21 \%)$ & $75 / 351(21 \%)$ & $17(22 \%)$ \\
\hline \multicolumn{4}{|l|}{ Prior residence } \\
\hline Home & $382(89 \%)$ & $308(87 \%)$ & $74(97 \%)$ \\
\hline Nursing home & $31(7 \%)$ & $30(8 \%)$ & $1(1.5 \%)$ \\
\hline Health-related institution & $6(1 \%)$ & $6(2 \%)$ & $0(0 \%)$ \\
\hline Others & $10(2 \%)$ & $9(3 \%)$ & $1(1.5 \%)$ \\
\hline \multicolumn{4}{|l|}{ Comorbidities } \\
\hline Hypertension & $225(52 \%)$ & $187(53 \%)$ & $38(50 \%)$ \\
\hline Diabetes mellitus & $161(37 \%)$ & $122(35 \%)$ & $39(51 \%)$ \\
\hline Coronary heart disease & $41(10 \%)$ & $36(10 \%)$ & $5(7 \%)$ \\
\hline Chronic heart failure & $28(6 \%)$ & $28(8 \%)$ & $0(0 \%)$ \\
\hline Chronic kidney disease & $61(14 \%)$ & $54(15 \%)$ & $7(9 \%)$ \\
\hline End-stage renal disease & $14(3 \%)$ & $12(3 \%)$ & $2(3 \%)$ \\
\hline Chronic respiratory disease & $51(12 \%)$ & $43(12 \%)$ & $8(10 \%)$ \\
\hline Chronic liver disease & $8(2 \%)$ & $6(2 \%)$ & $2(3 \%)$ \\
\hline Cerebrovascular accident & $57(13 \%)$ & $55(16 \%)$ & $2(3 \%)$ \\
\hline Immunosuppression (incl. HIV) & $26(6 \%)$ & $25(7 \%)$ & $1(1 \%)$ \\
\hline Sickle cell disease & $7(2 \%)$ & $5(1 \%)$ & $2(3 \%)$ \\
\hline Active cancer or haematological disease & $41(10 \%)$ & $39(11 \%)$ & $2(3 \%)$ \\
\hline \multicolumn{4}{|l|}{ Frailty } \\
\hline Frail & $170(40 \%)$ & $156 / 351(44 \%)$ & $14(18 \%)$ \\
\hline Not frail & $259(60 \%)$ & $197 / 351(56 \%)$ & $62(82 \%)$ \\
\hline \multicolumn{4}{|l|}{ Chief presenting symptoms } \\
\hline Fever & $265(62 \%)$ & $212(60 \%)$ & $53(70 \%)$ \\
\hline Cough & $266(62 \%)$ & $212(60 \%)$ & $54(71 \%)$ \\
\hline Dyspnoea & $184(43 \%)$ & $143(40 \%)$ & $41(54 \%)$ \\
\hline Fatigue & $101(23 \%)$ & $80(23 \%)$ & $21(28 \%)$ \\
\hline Lethargy & $82(19 \%)$ & $67(19 \%)$ & $15(20 \%)$ \\
\hline Days since symptom onset & $3.5(1-6)$ & $3(1-6)$ & $4.5(2-6)$ \\
\hline \multicolumn{4}{|l|}{ Reason for hospital admission } \\
\hline Medical (hypoxaemic respiratory failure) & $182(42 \%)$ & $125(35 \%)$ & $57(75 \%)$ \\
\hline Medical (other reasons) & $215(50 \%)$ & $203(57 \%)$ & $12(16 \%)$ \\
\hline Surgical or trauma & $29(7 \%)$ & $22(6 \%)$ & $7(9 \%)$ \\
\hline Elective & $3(1 \%)$ & $3(1 \%)$ & $0(0 \%)$ \\
\hline \multicolumn{4}{|l|}{ Initial chest $X$-ray } \\
\hline No abnormal findings & $95 / 405(23 \%)$ & $88 / 329(27 \%)$ & $7(9 \%)$ \\
\hline Diffuse opacities & $155 / 310(50 \%)$ & $105 / 241(44 \%)$ & $50 / 69(72 \%)$ \\
\hline Bilateral opacities & $178 / 310(57 \%)$ & $124 / 241(51 \%)$ & $54 / 69(78 \%)$ \\
\hline Pleural effusion & $42 / 310(13 \%)$ & $33 / 241(14 \%)$ & $9 / 69(13 \%)$ \\
\hline
\end{tabular}

Data are reported as median (IQR) or $n(\%)$, or $n / N(\%)$ when some data are missing. Abbreviations: ICU, Intensive Care Unit; BMI, body mass index (calculated as weight in kilograms divided by height in metres squared); ACCI, Age-adjusted Charlson Comorbidity Index; IMD, Index of Multiple Deprivation; HIV, human immunodeficiency virus. ${ }^{*}$ Ethnicity was missing in 38 patients (9\%). ${ }^{\dagger}$ BMI was missing in 133 patients (31\%). ${ }^{*}$ ACCI was missing for 28 patients $(6 \%)$. ${ }^{5} \mathrm{IMD}$ was missing in 2 patients $(<1 \%)$. ${ }^{5}$ Data on symptom onset were missing in $217 / 429(51 \%)$ of patients.

age (HR 1.07 per decade above 40 years; 95\% CI 1.04-1.09, $p<0.001$ ), male sex (HR 2.31; 95\% CI 1.52-3.50, $p<0.001$ ), raised admission CRP level (HR 1.03 per $10 \mathrm{mg} \cdot \mathrm{l}^{-1}$ increments above the upper normal limit of $5 \mathrm{mg} \cdot \mathrm{l}^{-1} ; 95 \% \mathrm{CI}$ $1.01-1.04, p=0.001$ ), history of CKD (HR 1.87; 95\% CI $1.21-2.89, \quad p=0.005)$, and dyspnoea as a presenting symptom (HR 1.88; 95\% CI 1.24-1.86, $p=0.005$ ). Ethnicity or level of deprivation was not associated with mortality in unadjusted or adjusted analyses. Stratification of the model by categories of age ( $\leq 60,>60$ years), sex, frailty, or ethnicity did not provide evidence of different baseline hazards. The effect of each included covariate, based on parametric modelling, is shown in Figure 2, with six examples of hypothetical patients. In the parametric model, male sex and 
TABle 2: Admission and most abnormal laboratory values for the study population, by location of admission.

\begin{tabular}{|c|c|c|c|c|c|c|c|}
\hline & \multicolumn{2}{|c|}{ Study population $(n=429)$} & \multicolumn{2}{|c|}{ Treated only on ward $(n=353)$} & \multicolumn{2}{|c|}{ Treated in ICU $(n=76)$} & \multirow{2}{*}{$\begin{array}{c}\text { Normal } \\
\text { range }\end{array}$} \\
\hline & Admission* & $\begin{array}{c}\text { Most } \\
\text { abnormal }^{\dagger}\end{array}$ & Admission* & $\begin{array}{c}\text { Most } \\
\text { abnormal }^{\dagger}\end{array}$ & Admission* & $\begin{array}{c}\text { Most } \\
\text { abnormal }^{\dagger}\end{array}$ & \\
\hline Haemoglobin $\left(\mathrm{g} \cdot \mathrm{l}^{-1}\right)$ & $128(110-141)$ & $110(87-125)$ & $127(110-140)$ & $113(97-129)$ & $134(114-148)$ & $78(68-94)$ & $115-155$ \\
\hline $\operatorname{WBC}\left(\times 10^{9} \cdot 1^{-1}\right)$ & $6.7(5.2-9.3)$ & $9.3(6.9-13.3)$ & $6.5(5.0-8.8)$ & $8.6(6.5-11.0)$ & $8.2(6.5-11.2)$ & $19.5(12.4-28.0)$ & $4.0-11.0$ \\
\hline $\begin{array}{l}\text { Neutrophils } \\
\left(\times 10^{9} \cdot 1^{-1}\right)\end{array}$ & $5.2(3.7-7.4)$ & $7.4(5.3-11.2)$ & $4.9(3.6-7.0)$ & $6.8(4.9-9.1)$ & $6.7(4.6-9.4)$ & $16.8(10.1-23.2)$ & $2.2-6.3$ \\
\hline $\begin{array}{l}\text { Lymphocytes } \\
\left(\times 10^{9} \cdot 1^{-1}\right)\end{array}$ & $1.0(0.7-1.4)$ & $0.8(0.5-1.1)$ & $1.0(0.7-1.4)$ & $0.8(0.6-1.1)$ & $1.0(0.8-1.3)$ & $0.7(0.5-0.8)$ & $1.3-4.0$ \\
\hline Platelets $\left(\times 10^{9} \cdot 1^{-1}\right)$ & $213(161-278)$ & $187(139-237)$ & $210(159-279)$ & $189(142-243)$ & $219(172-274)$ & $174(110-217)$ & $150-450$ \\
\hline $\mathrm{CRP}\left(\mathrm{mg} \cdot \mathrm{l}^{-1}\right)$ & $75(28-143)$ & $159(81-299)$ & $61(22-119)$ & $143(66-218)$ & $134(84-239)$ & $385(310-487)$ & $<5$ \\
\hline $\begin{array}{l}\text { Creatinine } \\
\left(\mu \mathrm{mol} \cdot l^{-1}\right)\end{array}$ & $92(68-130)$ & $102(77-207)$ & $91(68-128)$ & $97(74-143)$ & $96(69-150)$ & $324(108-533)$ & $45-120$ \\
\hline AST (IU.1 $\left.{ }^{-1}\right)$ & $43(29-69)$ & $70(44-122)$ & $39(27-59)$ & $59(39-92)$ & $63(39-102)$ & $196(104-396)$ & $10-50$ \\
\hline GGT $\left(\right.$ IU. $\left.1^{-1}\right)$ & $47(26-94)$ & $74(40-182)$ & $44(25-88)$ & $64(35-126)$ & $64(39-113)$ & $237(106-433)$ & $1-55$ \\
\hline Bilirubin $\left(\mu \mathrm{mol} \cdot \mathrm{l}^{-1}\right)$ & $8(5-11)$ & $10(7-15)$ & $7(5-11)$ & $9(6-13)$ & $9(6-13)$ & $15(10-23)$ & $3-20$ \\
\hline $\begin{array}{l}\text { Creatine kinase } \\
\left(\mathrm{U} \cdot \mathrm{l}^{-1}\right)\end{array}$ & $368(89-1553)$ & $\begin{array}{c}546 \\
(108-1870)\end{array}$ & $374(88-1628)$ & $311(78-1628)$ & $318(90-1363)$ & $773(168-2091)$ & $<150$ \\
\hline $\mathrm{LDH}\left(\mathrm{U} \cdot \mathrm{l}^{-1}\right)$ & $476(355-670)$ & $522(353-748)$ & $365(229-496)$ & $350(252-496)$ & $564(426-757)$ & $628(466-805)$ & $<240$ \\
\hline Troponin $T$ (ng. $\left.1^{-1}\right)$ & $25(11-54)$ & $35(15-85)$ & $25(11-60)$ & $27(11-65)$ & $26(10-51)$ & $51(22-174)$ & $<14$ \\
\hline Ferritin $\left(\mu \mathrm{g} \cdot \mathrm{l}^{-1}\right)$ & $914(298-1596)$ & $\begin{array}{c}1039 \\
(399-2374)\end{array}$ & $\begin{array}{c}666 \\
(258-1374)\end{array}$ & $\begin{array}{c}877 \\
(304-1512)\end{array}$ & $\begin{array}{c}1157 \\
(487-1777)\end{array}$ & $\begin{array}{c}1630 \\
(683-3423)\end{array}$ & $13-150$ \\
\hline D-Dimers (ng. $\left.{ }^{-1}\right)$ & $\begin{array}{c}1850 \\
(805-4090)\end{array}$ & $\begin{array}{c}1850 \\
(805-4090)\end{array}$ & $\begin{array}{c}1315 \\
(610-2470)\end{array}$ & $\begin{array}{c}1315 \\
(610-2470)\end{array}$ & $\begin{array}{c}3240 \\
(1160-6950)\end{array}$ & $\begin{array}{c}3240 \\
(1160-6950)\end{array}$ & $<500$ \\
\hline Lactate $\left(\mathrm{mmol} \cdot \mathrm{l}^{-1}\right)$ & $1.5(1.1-2.0)$ & $1.7(1.3-2.5)$ & $1.5(1.1-2.1)$ & $1.5(1.2-2.2)$ & $1.5(1.1-1.9)$ & $2.3(1.8-4.8)$ & $<2$ \\
\hline
\end{tabular}

Data are reported as median (IQR). Abbreviations: WBC, white blood cells; CRP, C-reactive protein; AST, aspartate aminotransferase; GGT, gammaglutamyl transferase; $\mathrm{LDH}$, lactate dehydrogenase. ${ }^{*}$ Admission laboratory tests were not performed or available for the following number of patients: haemoglobin $1 / 429(<1 \%)$, white blood cells $2 / 429(<1 \%)$, neutrophils $1 / 429(<1 \%)$, lymphocytes $1 / 429(<1 \%)$, platelets $8 / 429(2 \%)$, C-reactive protein $2 / 429$ $(<1 \%)$, creatinine $1 / 429(<1 \%)$, AST 35/429 (8\%), GGT 18/429 (4\%), bilirubin 5/429 (1\%), creatinine kinase 345/429 (80\%), LDH 363/429 (85\%), troponin 271/429 (63\%), ferritin 318/429 (74\%), D-dimers 309/429 (72\%), and lactate 86/429 (20\%). ${ }^{\dagger}$ Subsequent laboratory tests were not performed for the following number of patients: haemoglobin $8 / 429(2 \%)$, white blood cells $9 / 429(2 \%)$, neutrophils $7 / 429(2 \%)$, lymphocytes $9 / 429(2 \%)$, platelets $8 / 429(2 \%)$, C-reactive protein 6/429 (1\%), creatinine 6/429 (1\%), AST 25/429 (6\%), GGT 14/429 (3\%), bilirubin 9/429 (2\%), creatinine kinase 339/429 (79\%), LDH 356/429 (83\%), troponin 266/429 (62\%), ferritin 302/429 (70\%), D-dimers 309/429 (72\%), and lactate 115/429 (27\%).

dyspnoea on presentation had the largest impact on the hazard function. More details regarding the results of the parametric analysis are available in Supplementary File 3.

At the end of the 30-day follow-up, 15 of 353 ward patients (4\%) remained admitted in hospital and 12 of 270 (4\%) were readmitted after hospital discharge. Among all ICU patients, $13(17 \%)$ remained admitted in the ICU at the end of follow-up. Among the 50 patients discharged from the ICU, three (6\%) were readmitted to the ICU, $25(50 \%)$ were discharged from the hospital, three $(6 \%)$ died on the ward, and $22(44 \%)$ remained hospitalised on the ward.

During the study period, the hospital experienced a sudden surge in critically ill patients, and details regarding the number of hospital and ICU admissions are shown in Figure 3. Over a period of approximately six weeks, 20-40 patients were admitted daily to the hospital with COVID-19 and between five and ten of them required admission to the ICU for organ support. At the peak of the pandemic, both the hospital and ICUs experienced significant capacity strain, with $28 \%$ of the hospital capacity of 900 beds and $70 \%$ of the ICU surge capacity of 129 beds taken up by patients with COVID-19. This increased demand was supported by changes to the service described in Table 5. Major changes included anaesthetic cover for newly opened ICU beds, redeployment of the entire hospital workforce to support ICU, delivery of critical care in nonconventional areas like operating rooms, and the introduction of teams dedicated to tasks like prone positioning, communication with families, and tracheostomies. More details are available in Supplementary File 4.

\section{Discussion}

We describe data from 429 patients hospitalised with COVID-19 in an academic hospital in London UK, of which $76(18 \%)$ were treated in the ICU. We observed a disproportionate burden of COVID-19 hospitalisation and ICU admission in patients from an ethnic minority background, consistent with reports from similarly diverse areas [9] and the rest of the UK $[7,8,22]$. The range of clinical presentations was broad, similar to reports of the severe acute respiratory syndrome (SARS) and Middle East respiratory syndrome (MERS) [23, 24]. Respiratory involvement was very frequent, but not universally present. Hence, the reliance on the initial presence of respiratory failure or abnormal chest imaging results to make decisions regarding patient infectiousness and the requirement for isolation may be problematic.

Overall hospital mortality was identical to that reported in a recent large UK observational study [7], but patients 
TABLE 3: Clinical findings and outcomes during hospital admission.

\begin{tabular}{|c|c|c|c|}
\hline & Study population $(n=429)$ & Treated only on ward $(n=353)$ & Treated in ICU $(n=76)$ \\
\hline Fever on ward*† & $280 / 382(72 \%)$ & $252 / 349(72 \%)$ & $28 / 33(82 \%)$ \\
\hline Persistent hypoxia* ${ }^{\ddagger}$ & $105 / 386(27 \%)$ & $74 / 353(21 \%)$ & $31 / 33(94 \%)$ \\
\hline Noninvasive respiratory support ${ }^{* \S}$ & $17 / 386(4 \%)$ & $9 / 353(2 \%)$ & $8 / 33(24 \%)$ \\
\hline $\mathrm{SBP}<90 \mathrm{mmHg}$ for $>1$ hour* & $40 / 386(10 \%)$ & $37 / 353(10 \%)$ & $3 / 33(9 \%)$ \\
\hline Heart rate $>120 /$ minute for $>1$ hour* & $43 / 386(11 \%)$ & $38 / 353(11 \%)$ & $5 / 33(15 \%)$ \\
\hline AVPU score $\mathrm{V}$ or below for $>1$ hour* & $22 / 386(6 \%)$ & $19 / 353(5 \%)$ & $3 / 33(9 \%)$ \\
\hline NEWS2 score $>6^{*}$ & $195 / 378(52 \%)$ & $166 / 346(48 \%)$ & $31 / 32(91 \%)$ \\
\hline ICU outreach involvement* & $152 / 386(39 \%)$ & $87 / 353(25 \%)$ & $32 / 33(97 \%)$ \\
\hline COVID-19-specific drug treatment** & $7(2 \%)$ & $2 / 353(1 \%)$ & $5 / 76(7 \%)$ \\
\hline Subsequent chest X-ray & $198(46 \%)$ & $124 / 353(35 \%)$ & $74 / 76(97 \%)$ \\
\hline No abnormal findings & $21 / 198(11 \%)$ & $19 / 124(15 \%)$ & $2 / 74(3 \%)$ \\
\hline Diffuse opacities & $128 / 177(72 \%)$ & $64 / 105(61 \%)$ & $64 / 72(89 \%)$ \\
\hline Bilateral opacities & $141 / 177(80 \%)$ & $76 / 105(72 \%)$ & $65 / 72(90 \%)$ \\
\hline Pleural effusion & $46 / 177(26 \%)$ & $34 / 105(32 \%)$ & $12 / 72(17 \%)$ \\
\hline Pneumothorax & $3 / 177(2 \%)$ & $0(0 \%)$ & $3 / 72(4 \%)$ \\
\hline Chest CT & $59(14 \%)$ & $29 / 353(8 \%)$ & $30 / 76(39 \%)$ \\
\hline Diffuse GGOs or consolidation & $43 / 59(73 \%)$ & $15 / 29(52 \%)$ & $28 / 30(93 \%)$ \\
\hline Bilateral GGOs or consolidation & $47 / 59(80 \%)$ & $19 / 29(65 \%)$ & $28 / 30(93 \%)$ \\
\hline Pulmonary embolism & $15 / 59(25 \%)$ & $5 / 29(17 \%)$ & $10 / 30(33 \%)$ \\
\hline Organising pneumonia & $8 / 59(14 \%)$ & $4 / 29(14 \%)$ & $4 / 30(13 \%)$ \\
\hline Fibrosis & $7 / 59(12 \%)$ & $0(0 \%)$ & $7 / 30(23 \%)$ \\
\hline Echocardiogram & $57(13 \%)$ & $22(6 \%)$ & $35 / 76(46 \%)$ \\
\hline LV ejection fraction $<55 \%$ & $10 / 57(17 \%)$ & $7 / 22(32 \%)$ & $3 / 35(9 \%)$ \\
\hline Regional wall motion abnormalities & $3 / 57(5 \%)$ & $1 / 22(4 \%)$ & $2 / 35(6 \%)$ \\
\hline Pulmonary hypertension $^{\dagger \dagger}$ & $13 / 57(23 \%)$ & $4 / 22(18 \%)$ & $9 / 35(26 \%)$ \\
\hline TAPSE $<17$ millimetres & $2 / 57(3 \%)$ & $1 / 22(4 \%)$ & $1 / 35(3 \%)$ \\
\hline Pericardial effusion & $4 / 57(7 \%)$ & $4 / 22(18 \%)$ & $0(0 \%)$ \\
\hline Vascular scan & $23(5 \%)$ & $6(2 \%)$ & $17 / 76(22 \%)$ \\
\hline Deep vein thrombosis & $5 / 23(22 \%)$ & $2 / 6(33 \%)$ & $3 / 17(18 \%)$ \\
\hline Positive respiratory sample ${ }^{\ddagger}$ & $46(11 \%)$ & $8(2 \%)$ & $38 / 76(50 \%)$ \\
\hline Gram-positive organism & $4 / 42(9 \%)$ & $1 / 8(12 \%)$ & $3 / 38(8 \%)$ \\
\hline Gram-negative organism & $32 / 46(70 \%)$ & $3 / 8(37 \%)$ & $29 / 38(76 \%)$ \\
\hline Viral & $1 / 46(2 \%)$ & $1 / 8(12 \%)$ & $0(0 \%)$ \\
\hline Fungal & $19 / 46(41 \%)$ & $3 / 8(37 \%)$ & $16 / 28(42 \%)$ \\
\hline Positive blood sample & $57(13 \%)$ & $26(7 \%)$ & $31 / 76(41 \%)$ \\
\hline Gram-positive organism & $38 / 57(67 \%)$ & $19 / 26(73 \%)$ & $19 / 31(61 \%)$ \\
\hline Gram-negative organism & $15 / 57(26 \%)$ & $7 / 26(27 \%)$ & $8 / 31(26 \%)$ \\
\hline Viral & $13 / 57(29 \%)$ & $2 / 26(8 \%)$ & $11 / 31(35 \%)$ \\
\hline Fungal & $5 / 57(9 \%)$ & $0(0 \%)$ & $5 / 31(16 \%)$ \\
\hline Treatment limitations on ward* & $236 / 386(61 \%)$ & $232 / 353(66 \%)$ & $4 / 33(12 \%)$ \\
\hline Treatment escalation plan & $198 / 236(84 \%)$ & $196 / 232(84 \%)$ & $2 / 4(50 \%)$ \\
\hline Do-not-resuscitate order & $203 / 236(86 \%)$ & $200 / 232(86 \%)$ & $3 / 4(75 \%)$ \\
\hline Time to institution of TEP or DNR, days & $0(0-1)$ & $0(0-1)$ & $4(0-13.5)$ \\
\hline Deceased at hospital discharge, or at 30 days & $112(26 \%)$ & $83(23 \%)$ & $29(38 \%)$ \\
\hline Hospital length of stay, days ${ }^{\S \AA}$ & $10(4-22)$ & $8(4-18)$ & $20(12-35)$ \\
\hline Among survivors & $8(4-20)$ & $7(4-15)$ & $13(9-16)$ \\
\hline Among nonsurvivors & $10(4-24)$ & $8(4-20)$ & $34(16-38)$ \\
\hline
\end{tabular}

Data are reported as median (IQR) or $n(\%)$, or $n / N(\%)$ when some data are missing. Abbreviations: ICU, Intensive Care Unit; NEWS2, National Early Warning Score version 2; SBP, systolic blood pressure; $\mathrm{F}_{i} \mathrm{O}_{2}$, fraction of inspired oxygen; AVPU, "Alert, Verbal, Pain, Unresponsive" scale; COVID-19, coronavirus disease 19; CT, computed tomography; GGO, ground-glass opacities; LV, left ventricle; TAPSE, tricuspid annular plane systolic excursion. * These results apply to 386 patients: all 353 ward patients and 33/76 (43\%) ICU patients who were admitted to the ICU after being admitted to the ward for 12 hours or longer. ${ }^{\dagger}$ Defined as temperature of $38.0^{\circ} \mathrm{C}\left(100.4^{\circ} \mathrm{F}\right)$ or above. The temperature was missing in $4 / 386$ patients $(1 \%)$. ${ }^{*}$ Defined as oxygen requirement of more than 15 litres per minute ( or $\mathrm{F}_{i} \mathrm{O}_{2}$ of 0.6 or above), for one hour or longer. ${ }^{\S}$ Defined as the need for high-flow nasal oxygen, continuous positive airway pressure (CPAP), or noninvasive ventilation (NIV), for one hour or longer. 'NEWS2 score was missing for 8/386 patients (2\%). A NEWS2 score above 6 is considered a trigger for ICU outreach team assessment. ${ }^{* *}$ COVID-19-specific drug treatment included the administration of one of the following medications specifically for the purpose of treating COVID-19: human IL-1 receptor antagonists, chloroquine, hydroxychloroquine, dexamethasone, interferon-beta, lopinavir-ritonavir, remdesivir, tocilizumab, and mesenchymal stem cell therapy. ${ }^{\dagger \dagger}$ Defined as estimated right ventricular systolic pressure above $35 \mathrm{mmHg}$ or maximal tricuspid regurgitation velocity (TR $V_{\max }$ ) above 2.8 metres per second. ${ }^{* 1}$ Numbers refer to patients, not samples. ${ }^{\S \varsigma}$ For inpatients at study onset, length of stay was calculated from the start of the follow-up period (February 25, 2020). Those who remained admitted to the hospital at the end of the followup period (April 30,2020) were considered survivors, and length of stay was calculated until that date. 
TABle 4: Patient management and outcomes in the Intensive Care Unit.

\begin{tabular}{|c|c|}
\hline & Study population $(n=76)$ \\
\hline Fever in the ICU* & $57(78 \%)$ \\
\hline CPAP or NIV & $10(13 \%)$ \\
\hline Mechanical ventilation & $74(97 \%)$ \\
\hline Duration, days & $12(6-23)$ \\
\hline Renal replacement therapy & $43(57 \%)$ \\
\hline Duration, days & $11(4-17)$ \\
\hline Pharmacological circulatory support ${ }^{\dagger}$ & $61(80 \%)$ \\
\hline Duration, days & $8(4-14)$ \\
\hline \multicolumn{2}{|l|}{ SOFA score s $^{\ddagger}$} \\
\hline Day 1 (admission) & $14(13-16)$ \\
\hline Day 3 & $15(12-17)$ \\
\hline Day 7 & $15(13-17)$ \\
\hline Day 10 & $15(14-16)$ \\
\hline Day 14 & $14(12-17)$ \\
\hline \multicolumn{2}{|l|}{ Highest $\mathrm{F}_{i} \mathrm{O}_{2}{ }^{*}$} \\
\hline Day 1 (admission) & $0.6(0.5-0.8)$ \\
\hline Day 3 & $0.5(0.4-0.6)$ \\
\hline Day 7 & $0.5(0.4-0.7)$ \\
\hline Day 10 & $0.5(0.4-0.7)$ \\
\hline Day 14 & $0.4(0.3-0.7)$ \\
\hline \multicolumn{2}{|l|}{ Lowest $\mathrm{P}_{\mathrm{a}} \mathrm{O}_{2} / \mathrm{F}_{i} \mathrm{O}_{2}$ ratio, $\mathrm{kPa}^{\ddagger}$} \\
\hline Day 1 (admission) & $17.5(13.7-25)$ \\
\hline Day 3 & $19.2(14-24.7)$ \\
\hline Day 7 & $19(12.5-27.5)$ \\
\hline Day 10 & $20.5(15-29)$ \\
\hline Day 14 & $23.7(14-30.2)$ \\
\hline Rescue oxygenation strategies & $41(54 \%)$ \\
\hline Neuromuscular blocking drugs & $31(41 \%)$ \\
\hline Inhaled prostacyclin & $22(29 \%)$ \\
\hline Prone positioning & $19(25 \%)$ \\
\hline ECMO & $4(5 \%)$ \\
\hline Tracheostomy & $27(38 \%)$ \\
\hline Time to tracheostomy, days & $16.5(14-20)$ \\
\hline Treatment limitations in the ICU & $18(24 \%)$ \\
\hline Do-not-resuscitate (DNR) order & $15(20 \%)$ \\
\hline Time to DNR order, days & $6.5(4-13)$ \\
\hline Treatment escalation plan (TEP) & $16(21 \%)$ \\
\hline Time to TEP order, days & $7.5(3.5-12.5)$ \\
\hline Treatment withdrawal & $8(10 \%)$ \\
\hline Time to withdrawal, days & $12(4.5-18)$ \\
\hline $\begin{array}{l}\text { Deceased at ICU discharge, or at } 30 \\
\text { days }\end{array}$ & $26(34 \%)$ \\
\hline ICU length of stay, days ${ }^{\S}$ & $13(7-30)$ \\
\hline Among survivors & $19(9-32)$ \\
\hline Among nonsurvivors & $11(5-14)$ \\
\hline
\end{tabular}

Data are reported as median (IQR) or $n$ (\%). Abbreviations: CPAP, continuous positive airway pressure; NIV, noninvasive ventilation; SOFA, Sequential Organ Failure Assessment; $\mathrm{P}_{\mathrm{a}} \mathrm{O}_{2}$, partial pressure of oxygen in arterial blood; $\mathrm{F}_{i} \mathrm{O}_{2}$, fraction of inspired oxygen; ECMO, extracorporeal membrane oxygenation; ICU, Intensive Care Unit. * Defined as temperature of $38.3^{\circ} \mathrm{C}\left(101^{\circ} \mathrm{F}\right)$ or above. ${ }^{\dagger}$ Defined as the use of any vasopressor or inotropic drug. ${ }^{\ddagger}$ Data are missing for 1 patient $(1 \%)$. ${ }^{5}$ Those who remained admitted to the hospital at the end of the follow-up period (April 30, 2020) were considered survivors, and length of stay was calculated until that date.

admitted to the ward had higher morbidity and mortality than previously reported $[4,17,25]$. A significant proportion experienced clinical deterioration with cardiorespiratory compromise and AKI, meeting the criteria for critical care

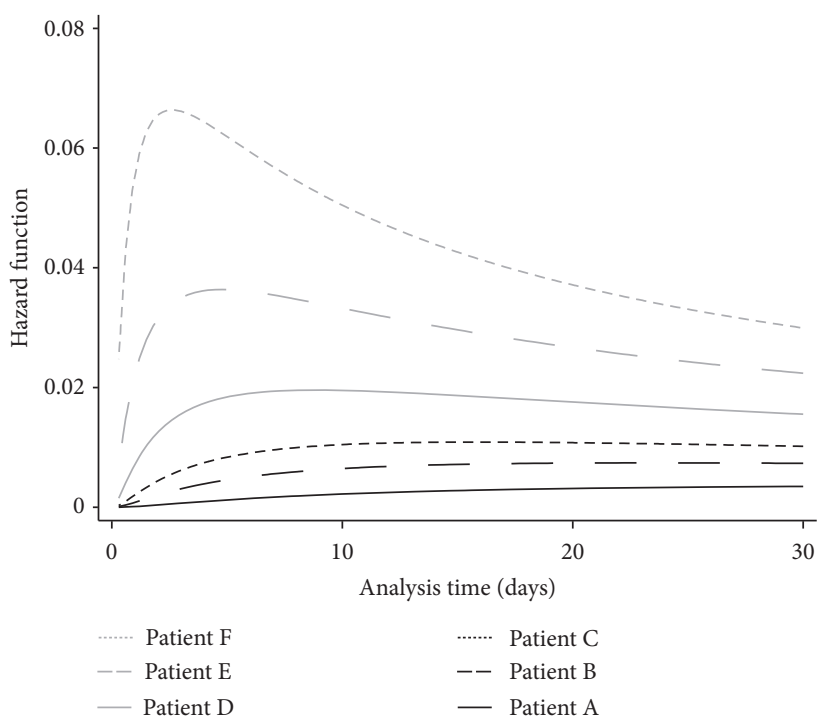

FIgURE 2: A plot of the primary outcome (hazard of death at hospital discharge or at 30 days) over time for six hypothetical patients, based on the parametric survival analysis. Patients' characteristics were selected to highlight the additional effect of individual risk factors on the hazard of death. Patient A represents a female 40-year-old patient with normal C-reactive protein levels $\left(\mathrm{CRP}<5 \mathrm{mg} \cdot \mathrm{l}^{-1}\right.$ ), no chronic kidney disease (CKD), and no dyspnoea on presentation. Patient B represents a male 40 -year-old patient with normal CRP, no CKD, and no dyspnoea. Patient $\mathrm{C}$ represents a male 65-year-old patient with normal CRP, no CKD, and no dyspnoea. Patient D represents a male 65-year-old patient with an abnormal CRP of $200 \mathrm{mg} \cdot \mathrm{l}^{-1}$, no CKD, and no dyspnoea. Patient E is similar to Patient D but has a history of CKD. Finally, Patient F represents a male 65-year-old patient with an abnormal CRP of $200 \mathrm{mg} \cdot \mathrm{l}^{-1}$, a history of CKD, and dyspnoea on presentation to the hospital.

outreach activation. Patients treated on the ward required considerable input from critical care services, with the ICU outreach team seeing almost four out of ten admitted patients. Similar patients may have been treated in the ICU in other described cohorts, and this may explain the difference in unadjusted mortality.

The contribution of the critical care outreach team, as evidenced by the number of patients seen, was crucial in our cohort; however, no comparison data exist. As part of their role, outreach teams commonly bridge the gap between ward and ICU by providing expertise, monitoring, and interventions outside the ICU environment and, importantly, by engaging in philosophy-of-care discussions with patients and their surrogate decision-makers [26]. In the context of many fixed constraints such as the number of ICU beds, the outreach service is a delivery model that greatly expands the availability of expertise and advanced treatments on the ward. Depending on the institutional context, however, outreach services require appropriate equipment and trained personnel such as doctors, nurses, and allied health professionals [26]. This has significant implications on staff planning and resource allocation during a pandemic.

The majority of ward-treated patients in our study had a decision to limit the escalation of therapy recorded during 


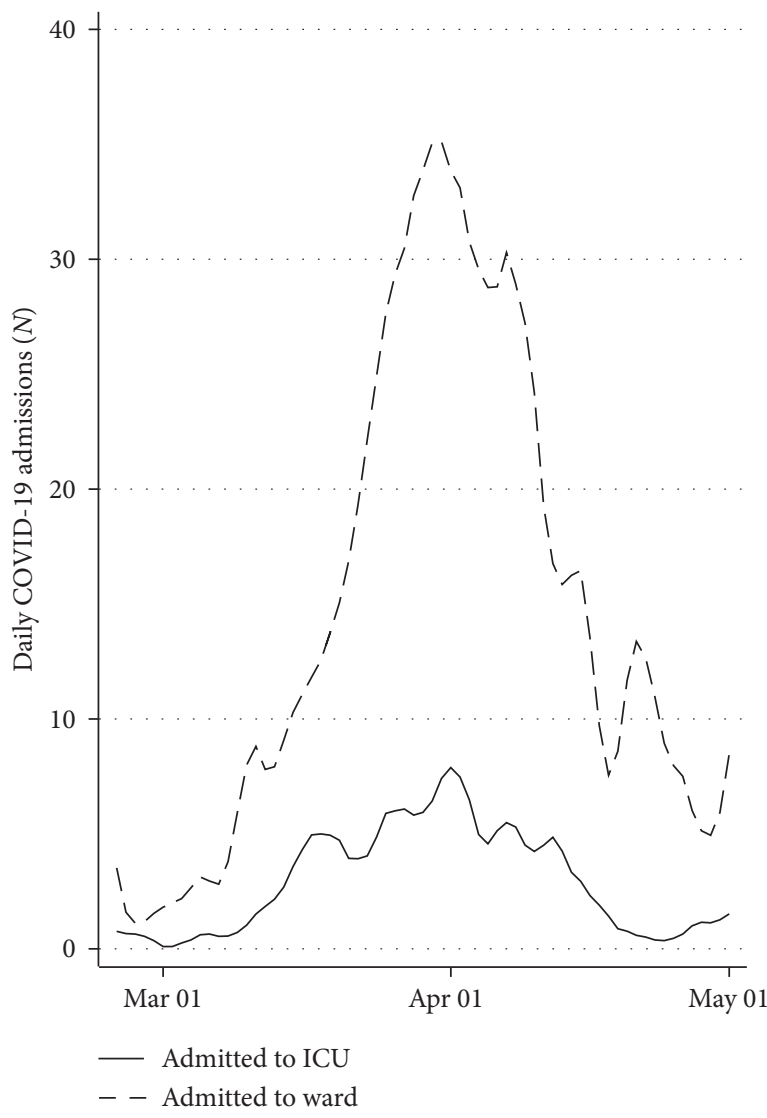

(a)

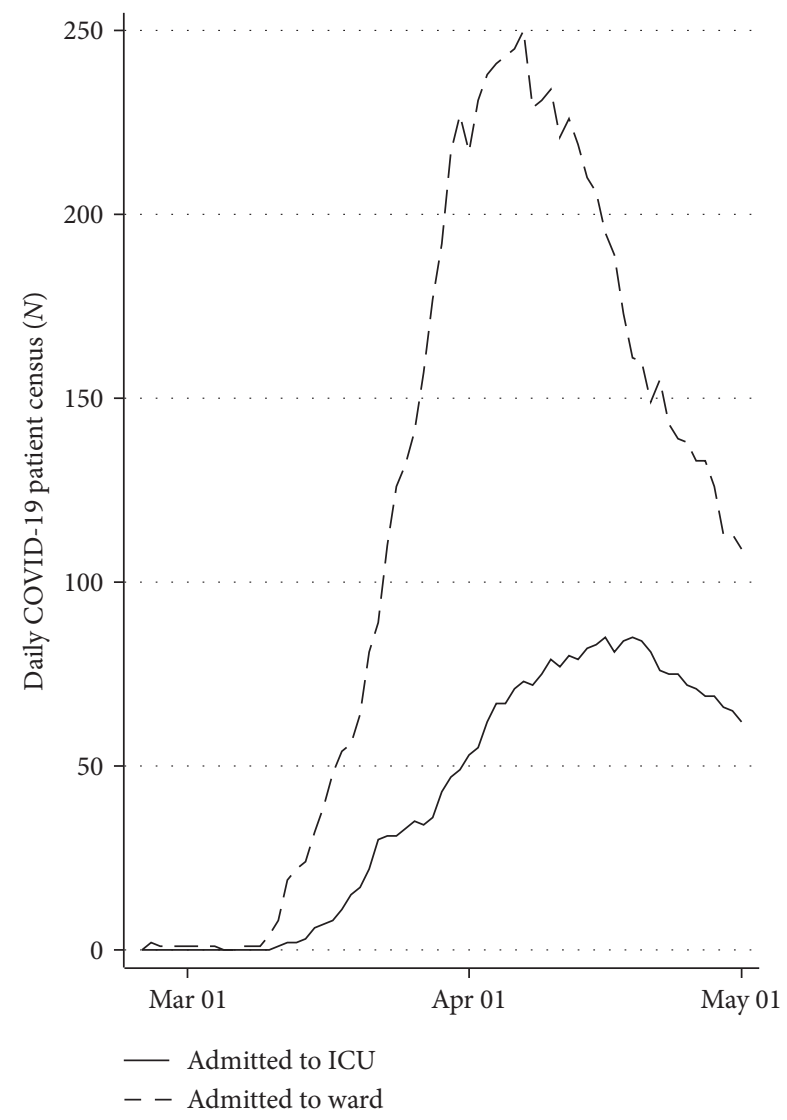

(b)

Figure 3: A plot of the surge of patients admitted with COVID-19 over the study period to the ward and the ICU. (a) Number of new patients admitted daily. (b) Overall number (census) of patients hospitalised with COVID-19.

TABLE 5: Examples of operational changes across different resource domains over the study period.

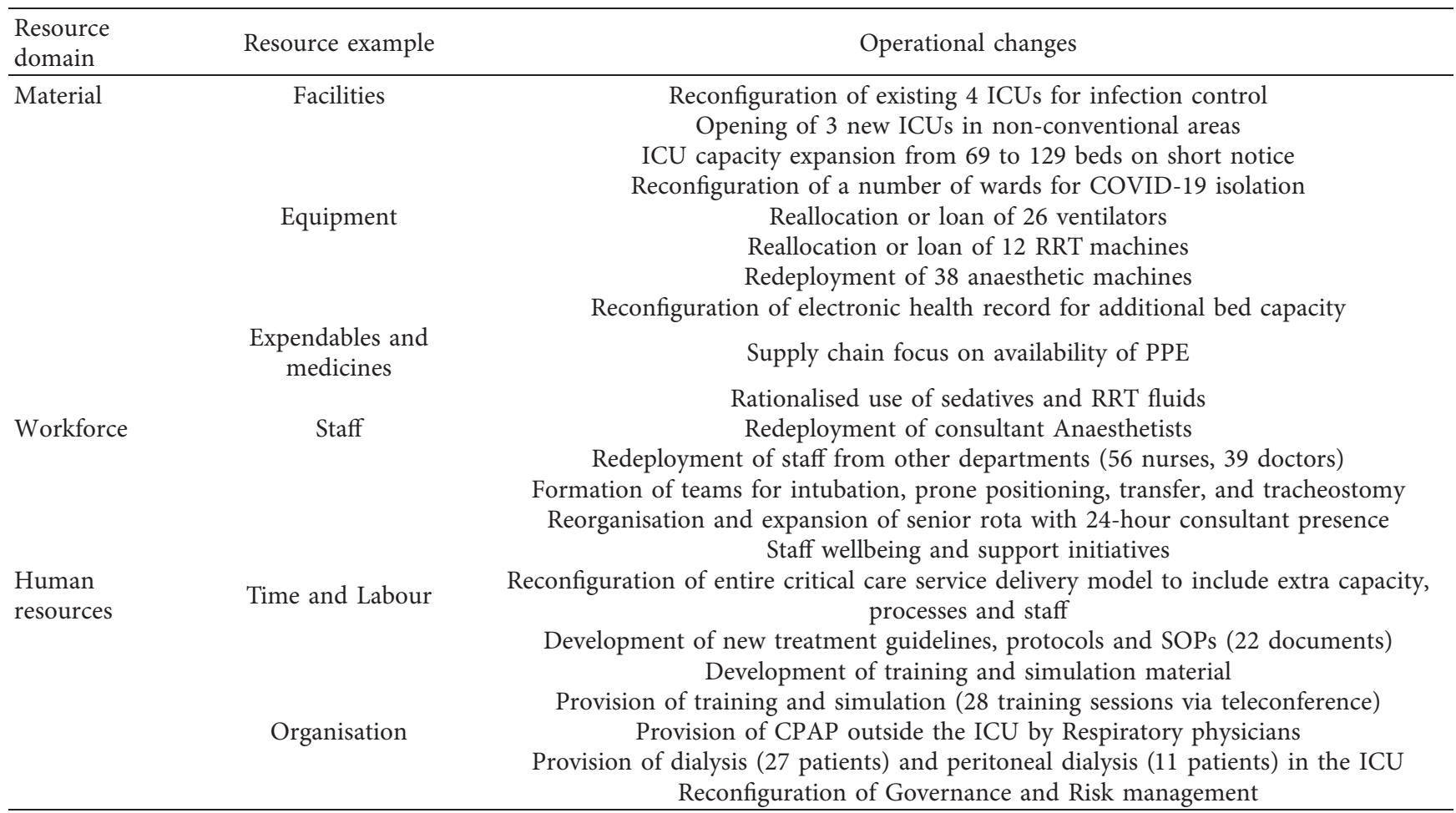


their admission, which represents a departure from previous practice. Most patients had treatment limitations placed upon admission. In addition to being clinically frail, serious comorbidities such as active malignancy and stroke were more common in these patients. Within the framework of generic national guidance regarding treatment escalation and ICU admission [27], we believe that the COVID-19 pandemic promoted more proactive communication about goals of care between physicians and their patients. In fact, Palliative Care Service in our institution faced a dramatic increase in ward referrals over the study period [28], but comparative data from other UK settings are lacking. The resource implications of delivering a comprehensive palliative care response during a pandemic are unknown but likely to be substantial [29-31].

Patients treated in the ICU had high morbidity and mortality. Few of them had trials of CPAP or NIV, and almost all required invasive mechanical ventilation, which differs from the experience in the UK [7] and other countries [10]. Pulmonary involvement was significant, with longer periods of mechanical ventilation and more frequent recourse to rescue oxygenation therapies than previously described [9]. Extrapulmonary organ involvement was also common and led to higher utilisation of pharmacological circulatory support and RRT than that reported across the UK [8] and in recent COVID-19 cohorts from Italy (27.8\%) [32], the USA (31\%) [9], and China (25\%) [33]. The high proportion of patients need organ support in the ICU could be explained by the fact that many patients with less severe organ failure were treated on the ward, with the aid of the outreach service. Similarly, the high incidence of significant extrapulmonary involvement supports the impression that critically ill COVID-19 patients are more similar to those with MERS [24], whereas SARS was a predominantly respiratory disease with single organ failure [34].

Hospital mortality in mechanically ventilated COVID19 patients was similar to that of unselected cohorts of severe adult respiratory distress syndrome (ARDS) patients who required ventilation $(46.1 \%)$, as well as that of mechanically ventilated patients with SARS (45\%) and MERS (52.4\%) [24, 34, 35]. In our survival analysis, we identified age, male sex, CKD, elevated CRP, and dyspnoea at presentation as important baseline prognostic factors associated with 30-day mortality after hospitalisation. These are broadly similar to patient characteristics described in reports from other countries [9, 36, 37] and the UK [7]. We did not, however, identify an independent association with ethnicity, which is also in line with findings from a recent large US cohort study [38]. Early identification of patient characteristics associated with poor clinical outcomes and higher resource utilisation is important when faced with the need to prioritise patients in the face of high demand for critical care services, both in the ICU and on the ward.

The COVID-19 pandemic led to a sudden surge in the overall number of critically ill patients across the hospital, as well as in the intensity and duration of treatments they required. This resulted in significant strain for the ICU in terms of capacity and service delivery. ICU bed capacity was effectively doubled; critically ill patients on the ward required frequent input by the outreach team, while patients in the ICU required prolonged mechanical ventilation and RRT, frequent rescue oxygenation therapies, and tracheostomy. To support this increased demand for critical care services, several operational changes were instituted, with support from Anaesthetic Department. These were based on local adaptation of lessons from previous infectious disease outbreaks and information from countries that had been already affected by COVID$19[2,3,39]$. The implementation of this service delivery model required the provision of a considerable amount of education and training. Also, these changes were implemented in a short time window and required collaboration across the entire hospital. Our lesson from this process is that clinicians and health administrators need to consider a rapidly scalable model, as health systems could easily become overwhelmed, and our experience is directly comparable to that from other, similarly affected, metropolitan areas [40].

Our study has several strengths. First, it includes a clearly defined cohort with near-complete 30-day outcomes, thus minimising selection bias. Second, it provides a granular clinical description of patient trajectories with previously unknown information regarding ICU outreach involvement and palliative care practice. Third, the inclusion of patients treated both on the ward and in the ICU offers a more complete picture of the organ dysfunctions experienced and the corresponding resource implications. Finally, we provide unique details regarding hospital and ICU capacity strain and service reorganisation.

Our study results must, however, be viewed in light of its methodological limitations. The prospective design relied on abstracting data from the EHR and is susceptible to missing data and recording bias. We identified a number of factors associated with mortality but do not imply any causal relationships. Finally, this study was performed in a single centre in the UK and this may limit its generalisability to other institutional contexts.

\section{Conclusions}

Our study identified age, sex, CKD, baseline CRP, and respiratory involvement as factors associated with hospital mortality in COVID-19 patients admitted to a large academic hospital in London, UK. Patients treated on the ward and in the ICU had a significant extrapulmonary disease, frequent treatment limitations, and a high burden of mortality. Medical wards caring for COVID-19 patients experienced a substantially increased workload and required frequent ICU outreach input. In order to provide effective care under pandemic surge conditions, significant reorganisation took place within the hospital. As a result, when planning an effective response to support patients with COVID-19, clinicians and health administrators should consider the need for additional critical care resources in the ICU, as well as the wider hospital. 


\section{Abbreviations}

\begin{tabular}{|c|c|}
\hline ACCI: & Age-adjusted Charlson Comorbidity Index \\
\hline AHP: & Allied health professions \\
\hline AKI: & Acute kidney injury \\
\hline ARDS: & Adult respiratory distress syndrome \\
\hline AST: & Aspartate aminotransferase \\
\hline AVPU: & "Alert, verbal, pain, unresponsive" scale \\
\hline BMI: & Body mass index \\
\hline CKD: & Chronic kidney disease \\
\hline COVID-19: & Coronavirus disease-19 \\
\hline CPAP: & Continuous positive airway pressure \\
\hline CRP: & C-reactive protein \\
\hline CT: & Computed tomography \\
\hline DNR: & Do-not-resuscitate \\
\hline ECMO: & Extracorporeal membrane oxygenation \\
\hline EHR: & Electronic health record \\
\hline $\mathrm{F}_{i} \mathrm{O}_{2}$ & Fraction of inspired oxygen \\
\hline GGO: & Ground-glass opacities \\
\hline GGT: & Gamma-glutamyl transferase \\
\hline HR: & Hazard ratio \\
\hline HIV: & Human immunodeficiency virus \\
\hline ICU: & Intensive Care Unit \\
\hline IMD: & Index of Multiple Deprivation \\
\hline IQR: & Interquartile range \\
\hline LDH: & Lactate dehydrogenase \\
\hline LV: & Left ventricle \\
\hline MERS: & Middle East respiratory syndrome \\
\hline NEWS2: & National Early Warning Score version 2 \\
\hline NIV: & Noninvasive ventilation \\
\hline $\mathrm{P}_{\mathrm{a}} \mathrm{O}_{2}$ : & Arterial partial pressure of oxygen \\
\hline PE: & Pulmonary embolism \\
\hline PFR: & $\mathrm{P}_{\mathrm{a}} \mathrm{O}_{2} / \mathrm{F}_{\mathrm{i}} \mathrm{O}_{2}$ ratio \\
\hline PPE: & Personal protective equipment \\
\hline RRT: & Renal replacement therapy \\
\hline RT-PCR: & $\begin{array}{l}\text { Reverse-transcriptase polymerase chain } \\
\text { reaction }\end{array}$ \\
\hline $\begin{array}{l}\text { SARS-CoV- } \\
2:\end{array}$ & $\begin{array}{l}\text { Severe acute respiratory syndrome } \\
\text { coronavirus- } 2\end{array}$ \\
\hline SARS: & Severe acute respiratory syndrome \\
\hline SBP: & Systolic blood pressure \\
\hline SOFA: & Sequential Organ Failure Assessment \\
\hline SOP: & Standard operating procedure \\
\hline TEP: & Treatment escalation plan \\
\hline UK: & United Kingdom \\
\hline USA: & United States of America \\
\hline WBC: & White blood cells. \\
\hline
\end{tabular}

\section{Data Availability}

The dataset generated and analysed during the current study is not publicly available due to Institutional Review Board approval restrictions.

\section{Disclosure}

This paper has been published as a preprint and is available at the following site: https://www.medrxiv.org/content/10. $1101 / 2020.07 .16 .20155069 v 1$. This research did not receive any funding and was performed as part of the authors' employment with King's College Hospital NHS Foundation Trust.

\section{Conflicts of Interest}

The authors declare that they have no conflicts of interest.

\section{Authors' Contributions}

SV and RM conceived the study and its design, had full access to the data, and take responsibility for the integrity of the data and accuracy of the analysis. SV, AW, VM, SC, CLS, JP, KL, TP, CS, KA, BA, RB, FJ, SAH, WB, and RM extracted and entered the data. SV and RM contributed to data analyses. SV, AW, VM, SC, CLS, JP, KL, WB, and RM contributed to data interpretation. SV and RM drafted the manuscript. All authors critically revised the drafted manuscript and approved the submitted manuscript.

\section{Acknowledgments}

We thank Professor Alistair McGuire (Department of Health Policy, London School of Economics) for his valuable help in the preparation of the manuscript. We also thank King's College Hospital Business Intelligence Unit for providing us with heatmap images of the pandemic evolution in the area. We thank "Easy Mobile Forms Software, The DataDyne Group, LLC" for providing 2 months of free access to their data collection tool Magpi. Finally, we would like to thank our patients and fellow health-care workers for providing outstanding patient care at considerable personal risk.

\section{Supplementary Materials}

Supplementary File 1. File name: Supplementary_file_1.pdf. Title: institutional setting. Description: details regarding the institutional setting of King's College Hospital and pandemic surge in the catchment area, including its temporal evolution with the use of heat maps. Supplementary File 2. File name: Supplementary_file_2.pdf. Title: definition of variables. Description: details regarding the definitions of variables used in the study. Supplementary File 3. File name: Supplementary_file_3.pdf. Title: statistical methods. Description: details regarding the statistical approach including the selection of variables and models. Description of the parametric regression equation. Additional figure describes the effect of each variable on the primary outcome of hospital mortality. Supplementary File 4. File name: Supplementary_file_4.pdf. Title: institutional surge planning and resource implications. Description: details regarding the reconfiguration of services implemented during the pandemic surge and the resources required. (Supplementary Materials)

\section{References}

[1] World Health Organization, World Health Organization Situation Report-133 June 1, https://www.who.int/docs/ default-source/coronaviruse/situation-reports/20200601covid-19-sitrep-133.pdf?sfvrsn=9a56f2ac_4, World Health 
Organization, Geneva, Switzerland, 2020, https://www.who. int/docs/default-source/coronaviruse/situation-reports/ 20200601-covid-19-sitrep-133.pdf?sfvrsn=9a56f2ac_4.

[2] Z. Wu and J. M. McGoogan, "Characteristics of and important lessons from the coronavirus disease 2019 (COVID19) outbreak in China," JAMA, vol. 323, no. 13, pp. 1239-1242, 2020.

[3] G. Grasselli, A. Pesenti, and M. Cecconi, "Critical care utilization for the COVID-19 outbreak in Lombardy, Italy," JAMA, vol. 323, no. 16, pp. 1545-1546, 2020.

[4] S. Richardson, J. S. Hirsch, M. Narasimhan et al., "Presenting characteristics, comorbidities, and outcomes among 5700 patients hospitalized with COVID-19 in the New York city area," JAMA, vol. 323, no. 20, pp. 2052-2059, 2020.

[5] Public Health England, "Daily UK COVID-19 cases update," Public Health England, London, UK, 2020, https:// coronavirus.data.gov.uk.

[6] R. A. Armstrong, A. D. Kane, and T. M. Cook, "Outcomes from intensive care in patients with COVID—19: a systematic review and meta-analysis of observational studies," Anaesthesia, vol. 75, no. 10, pp. 1340-1349, 2020.

[7] A. B. Docherty, E. M. Harrison, C. A. Green et al., "Features of 20133 UK patients in hospital with COVID-19 using the ISARIC WHO clinical characterisation protocol: prospective observational cohort study," BMJ, vol. 369, Article ID m1985, 2020.

[8] Intensive Care National Audit \& Research Centre, "ICNARC report on COVID-19 in critical care," https://www.icnarc.org/ Our-Audit/Audits/Cmp/Reports, Intensive Care National Audit \& Research Centre, London, UK, 2020, https://www. icnarc.org/Our-Audit/Audits/Cmp/Reports.

[9] M. J. Cummings, M. R. Baldwin, D. Abrams et al., "Epidemiology, clinical course, and outcomes of critically ill adults with COVID-19 in New York City: a prospective cohort study," The Lancet, vol. 395, no. 10239, pp. 1763-1770, 2020.

[10] G. Grasselli, A. Zangrillo, A. Zanella et al., "Baseline characteristics and outcomes of 1591 patients infected with SARSCoV-2 admitted to ICUs of the Lombardy region, Italy," JAMA, vol. 323, no. 16, pp. 1574-1581, 2020.

[11] World Health Organization, Clinical Management of Severe Acute Respiratory Infection when COVID-19 Is Suspected (v1.2), World Health Organization, Geneva, Switzerland, 2020, https://www.who.int/publications-detail/clinicalmanagement-of-severe-acute-respiratory-infection-whennovel-coronavirus-(ncov)-infection-is-suspected.

[12] E. von Elm, D. G. Altman, M. Egger et al., "The strengthening the reporting of observational studies in Epidemiology (STROBE) statement: guidelines for reporting observational studies," International Journal of Surgery, vol. 12, no. 12, pp. 1495-1499, 2014.

[13] Magpi, Easy Mobile Forms Software, The DataDyne Group, LLC, Washington, DC, USA, 2020.

[14] Ministry of Housing Communities \& Local Government English indices of deprivation 2019. https://www.gov.uk/ government/statistics/english-indices-of-deprivation-2019. 2020.

[15] G. B. Smith, O. C. Redfern, M. A. Pimentel et al., "The National Early Warning Score 2 (NEWS2)," Clinical Medicine, vol. 19, no. 3, p. 260, 2019.

[16] J.-L. Vincent, R. Moreno, J. Takala et al., "The SOFA (Sepsisrelated Organ Failure Assessment) score to describe organ dysfunction/failure," Intensive Care Medicine, vol. 22, no. 7, pp. 707-710, 1996.
[17] L. C. Myers, S. M. Parodi, G. J. Escobar, and V. X. Liu, "Characteristics of hospitalized adults with COVID-19 in an integrated health care system in California," JAMA, vol. 323, no. 21, pp. 2195-2198, 2020.

[18] K. Rockwood, X. Song, and C. MacKnight, "A global clinical measure of fitness and frailty in elderly people," Canadian Medical Association Journal, vol. 173, no. 5, pp. 489-495, 2005.

[19] J. Muscedere, B. Waters, A. Varambally et al., "The impact of frailty on intensive care unit outcomes: a systematic review and meta-analysis," Intensive Care Medicine, vol. 43, no. 8, pp. 1105-1122, 2017.

[20] L. Obolensky, T. Clark, G. Matthew, and M. Mercer, "A patient and relative centred evaluation of treatment escalation plans: a replacement for the do-not-resuscitate process," Journal of Medical Ethics, vol. 36, no. 9, pp. 518-520, 2010.

[21] KDIGO, "Section 2: AKI definition," Kidney International Supplements, vol. 2, no. 1, pp. 19-36, 2012.

[22] C. Lassale, B. Gaye, M. Hamer, C. R. Gale, and G. D. Batty, "Ethnic disparities in hospitalisation for COVID-19 in England: the role of socioeconomic factors, mental health, and inflammatory and pro-inflammatory factors in a communitybased cohort study," Brain, Behavior, and Immunity, vol. 88, pp. 44-49, 2020.

[23] J. S. M. Peiris, K. Y. Yuen, A. D. M. E. Osterhaus, and K. Stöhr, "The severe acute respiratory syndrome," New England Journal of Medicine, vol. 349, no. 25, pp. 2431-2441, 2003.

[24] Y. M. Arabi, A. Al-Omari, Y. Mandourah et al., "Critically ill patients with the Middle East respiratory syndrome," Critical Care Medicine, vol. 45, no. 10, pp. 1683-1695, 2017.

[25] W.-J. Guan, Z.-Y. Ni, Y. Hu et al., "Clinical characteristics of coronavirus disease 2019 in China," New England Journal of Medicine, vol. 382, no. 18, pp. 1708-1720, 2020.

[26] D. A. Jones, M. A. DeVita, and R. Bellomo, "Rapid-response teams," New England Journal of Medicine, vol. 365, no. 2, pp. 139-146, 2011.

[27] Royal College of Physicians, Ethical Dimensions of COVID-19 for Frontline Staff, https://www.rcplondon.ac.uk/news/ ethical-guidance-published-frontline-staff-dealingpandemic, Royal College of Physicians, London, UK, 2020, https://www.rcplondon.ac.uk/news/ethical-guidancepublished-frontline-staff-dealing-pandemic.

[28] N. Lovell, M. Maddocks, S. N. Etkind et al., "Characteristics, symptom management, and outcomes of 101 patients with COVID-19 referred for hospital palliative care," Journal of Pain and Symptom Management, vol. 60, no. 1, pp. e77-e81, 2020.

[29] C. L. Sprung, G. M. Joynt, M. D. Christian, R. D. Truog, J. Rello, and J. L. Nates, "Adult ICU triage during the coronavirus disease 2019 pandemic: who will live and who will die? Recommendations to improve survival*," Critical Care Medicine, vol. 48, no. 8, pp. 1196-1202, 2020.

[30] P. May, C. Normand, J. B. Cassel et al., "Economics of palliative care for hospitalized adults with serious illness," JAMA Internal Medicine, vol. 178, no. 6, pp. 820-829, 2018.

[31] J. Fausto, L. Hirano, D. Lam et al., "Creating a palliative care inpatient response plan for COVID-19-the UW medicine experience," Journal of Pain and Symptom Management, vol. 60, no. 1, pp. e21-e26, 2020.

[32] V. Fanelli, M. Fiorentino, V. Cantaluppi et al., "Acute kidney injury in SARS-CoV-2 infected patients," Critical Care, vol. 24, no. 1, p. 155, 2020.

[33] X. Yang, Y. Yu, J. Xu et al., "Clinical course and outcomes of critically ill patients with SARS-CoV-2 pneumonia in Wuhan, China: a single-centered, retrospective, observational study," 
The Lancet Respiratory Medicine, vol. 8, no. 5, pp. 475-481, 2020.

[34] R. A. Fowler, S. E. Lapinsky, and D Hallett, "Critically ill patients with severe acute respiratory syndrome," JAMA, vol. 290, no. 3, pp. 367-373, 2003.

[35] G. Bellani, J. G. Laffey, T. Pham et al., "Epidemiology, patterns of care, and mortality for patients with acute respiratory distress syndrome in intensive care units in 50 countries," JAMA, vol. 315, no. 8, pp. 788-800, 2016.

[36] W. Liang, H. Liang, L. Ou et al., "Development and validation of a clinical risk score to predict the occurrence of critical illness in hospitalized patients with COVID-19," JAMA Internal Medicine, vol. 180, no. 8, pp. 1081-1089, 2020.

[37] C. Wu, X. Chen, Y. Cai et al., "Risk factors associated with acute respiratory distress syndrome and death in patients with coronavirus disease 2019 pneumonia in Wuhan, China," JAMA Internal Medicine, vol. 180, no. 7, pp. 934-943, 2020.

[38] E. G. Price-Haywood, J. Burton, D. Fort, and L. Seoane, "Hospitalization and mortality among black patients and white patients with COVID-19," New England Journal of Medicine, vol. 382, no. 26, pp. 2534-2543, 2020.

[39] T. Kain and R. Fowler, "Preparing intensive care for the next pandemic influenza," Critical Care, vol. 23, no. 1, p. 337, 2019.

[40] K. M. Griffin, M. G. Karas, N. S. Ivascu, and L. Lief, "Hospital preparedness for COVID-19: a practical guide from a critical care perspective," American Journal of Respiratory and Critical Care Medicine, vol. 201, no. 11, pp. 1337-1344, 2020. 